\title{
LOBECTOMIA TEMPORAL NO INFARTO CEREBRAL COM EFEITO DE MASSA
}

\author{
LUIS FERNANDO MARTINS *, VALTER DA COSTA *, JOAQUIM DA COSTA *, \\ SEBASTIAO EURICO DE MELO-SOUZA **
}

\begin{abstract}
RESUMO - Uma complicação frequentemente fatal do infarto isquêmico extenso é o desenvolvimento de efeito de massa por edema. O componente de «swelling» pode ser combatido, mas o edema isquêmico citotóxicu dos primeiros dias não responde ao tratamento clínico. Este estudo mostra o resultado da abordagem cirúrgica em casos com deterioraça clínica e grande efeito de massa à tomografia computadorizada, nos quais se procedeu a lobectomia temporal do lado do infarto, com finalidade descompressiva. Dos 8 casos, 6 sobreviveram com efeito benéfico quase imediato à cirurgia. Apesar da pequena amostra e de não haver um controle rigoroso de amostragem, os autores sugerem que a técnica é de valor na conduta de tais casos e recomendam estudos posteriores melhor controlados.
\end{abstract}

PALAVRAS-CHAVE: acidente vascular encefálico, infarto cerebral, herniação do lobo temporal, lobectomia temporal.

\section{Temporal lobectomy in ischemic infarction with mass effect.}

SUMMARY - Cerebral edema secondary to ischemia can threaten life, mainly due to frequent failure of medical management. Imminent herniation of the temporal lobe can be timely detected by clinical signs and CT-scan. Eight patients (4M, 4F; 48-74 years, mean 62) with ischemic stroke and imminent herniation, were surgically decompressed by a standard temporal lobectomy as described by olivier for temporal lobe epilepsy. Six patients survived, two died and were considered failure of the procedure, probably due to late surgical indication. In conclusion, temporal lobectomy is life-saving for patients with large ischemic cerebral infarction with mass effect and deteriorating signs of brain stem compression.

KEY WORDS: stroke, brain infarction, temporal lobe herniation, temporal lobectomy.

No infarto cerebral isquêmico desenvolve-se edema tipo citotóxico nos primeiros dias após sua instalação 13 . Em alguns casos, pode também complicar-se com "swelling" devido a hipoxemia e consequente vasodilatação cerebral. Se o infarto é extenso, pode-se esperar que o edema, principalmente se associado a "swelling", aumente o volume da lesão a ponto de provocar desvios e compressões. A deterioração clínica ocorre pelo comprometimento da função do tronco cerebral ${ }^{9}$ e não são poucos os casos que têm evolução fatal 13 . O tratamento clínico é capaz apenas de manter o paciente em boas condiçóes gerais de ventilação e circulação, o que não é suficiente para impedir o desenvolvimento do edema embora necessário para tentar evitar o "swelling". Entretanto, não é sempre que se instala uma abordagem adequada que, por vezes, é considerada agressiva para um paciente que está aparentemente bem. $O$ edema isquêmico não responde a qualquer terapêutica disponivel, aguardando-se novas tendências, com a utilização de bloqueadores de radicais livres e das excitotoxinas, entre outros 4 . Naqueles casos de piora progressiva, cuja causa é o efeito de massa produzido pela ex-

Trabalho realizado no Instituto de Neurologia de Goiânia: * Neurocirurgião; ** Neurologista, Coordenador de Ensino e Pesquisa. Aceite: 27-junho-1992.

Dr. Sebastiăo Eurico de Melo-Souza - Caixa Postal 1181 - 74001-970 Goiânia GO - Brasil. 
pansão do infarto, pode-se tentar algum tipo de cirurgia descompressiva, em último recurso $3,5,6,7,10$.

Aproveitando a experiência da equipe de cirurgia de epilepsia do Instituto de Neurologia de Goiânia (ING), temos realizado a lobectomia temporal em casos de infarto cerebral massivo. A experiência com os primeiros 8 casos é o motivo da apresentação deste estudo.

\section{MATERIAL E METODOS}

O material é constituído de 8 pacientes internados no ING entre setembro-1990 e dezembro-1991, sendo 4 homens e 4 mulheres com idades variando de 48 a 74 anos (média 62 anos).

A técnica cirúrgica foi a mesma descrita por Olivier 10 para labectomia temporal: sob anestesia geral, faz-se extensa craniotomia fronto-temporal, expondo-se a região anteromedial do lobo temporál; procede-se a corticectomia inicial no sentido do giro temporal superior ao giro fusiforme. Depois, no sentido vertical, através do corno temporal do ventrículo lateral é concluida a ressecçâo das porções mesiais do uncus, giro para-hipocampal, amígdala e porção anterior do hipocsampo.

\section{CASUISTICA}

Caso 1 - SRN, 67 anos, branco, masculino. Paciente hipertenso crônico admitido na UTI em 08-09-90, 1 hora após ter sido encontrado caído dentro de casa, com paralisia do lado esquerdo (E) do corpo. Exame: PA: 16x8, desvio ocullar para a direita (D), hemiplegia E, paralisia facial central E, confuso, pupilas discóricas (cirurgias prévias), nuca livre, verbalizando respostas. Exame cardiológico normal. Realizada tomografia computadorizada do crânio (TCC), que foi normal. Medicado com dexametasona, ticlopidina e nimodipina. No dia seguinte estava torporoso, reagindo à dor do lado $\mathrm{D}$, hemiplegia $\mathrm{E}$, carótidas normais, nuca livre, pupilas discóricas. Introduzido Rheomacrodex, $500 \mathrm{ml}$ em dose única. No dia 10-09-90 0 paciente estava torporoso, atendendo às solicitaç̃es e esbocando alguns movimentos do lado $\mathbf{E}$ do corpo; Rheomacrodex 250ml. Em 11-09-90: comatoso, respondendo mal aos estímulos verbais, hemiplegia E, pupilas discóricas, respiração tipo Cheyne-stokes, demonstrando grande piora do quadro clínico. Repetida TCC, com extenso infarto fronto-têmporo-parietal com efeito de massa importante, à D. O paciente foi operado às 9:30 horas: lobectomia temporal D. No dia seguinte o paciente estava mais desperto, atendendo às solicitações verbais, verbalizandoł com dificuldade, hemiplegia $\mathrm{E}$ inalterada e sinais vitais estáveis. Melhora gradual do nível de consciência até a alta, do CTI em 19-09 e hospitalar em 26-09-90, consciente, lúcido e com hemiplegia $\mathbf{E}$ maciça.

Caso 2 - LAC, 66 anos, branca, feminina. Admitida em 14-10-90 após quadro súbito de hemiplegia D completa e perda da fala, 2 horas após o icto. Exame: PA: 16x9, sem rigidez de nuca, consciente, hemiplegia $D$ completa e macica, afasia. TCC: discreta hipodensidade fronto-parietal E e pequeno efeito de massa (Fig 1A), Exame cardiológico normal. Medicada com Rheomacrodex e pentoxifilina. No dia seguinte estava torporosa, mantendo os olhos abertos mas não atendendo às solicitações, hemiplegia $D$ maciça, nuca livre. No dia 16 estava pior, comatosa, com secreção brônquica abundante, respiração tipo Cheyne-Stokes, sendo transferida para o CTI. A tarde estava com anisocoria (pupila $\mathbf{E}$ maior que a $D$ ) e reação de hipertonia em extensão à $D$. TCC: extenso infarto fronto-têmporo-parietal $E$ e grande efeito de massa (Fig. 1B). Realizada lobectomia temporal às 19:00 horas deste dia. No dia seguinte mantinha-se torporosa, reagindo e despertando aos estímulos álgicos, anisocoria com pupila E maior que a D. Manteve-se ainda torporosa nos dias subsequentes, tendo sido submetida a traqueostomia no dia 22-10. Melhora lenta e progressiva, recebendo alta do CTI em 26-10 e hospitalar em 08-11-90, consciente, afásica e com hemiplegia $D$ maciça.

Casso 3 - HND, 68 anos, branca, feminina. Admitida na UTI em 09-12-90, 18 horas após o icto com hemiplegia D e afasia. Exame: PA: 18x10, consciente, não obedece comandos, isocórica, fotorreagente, hemiplegia $D$ maciça e completa, afasia. TCC: infarto têmporo-parietal E, com discreto efeito de massa. Medicada com Rheomacrodex. No dia seguinte mantinha-se ainda estável, um pouco sonolenta, atendendo a comandos verbais. No dia 11, torporosa com reação de despertar prejudicada, isocórica e fotorreagente. As 16:00 horas entrou em coma, com respiração tipo Cheyne-Stokes, ainda isocórica e fotorreagente. TCC: extenso infarto 
fronto-têmpero-parietal e grande efeito de massa. Às 21:00 horas submetida a lobectomia temporal E. No dia seguinte estava com respiracão espontânea, despertando tao estímulo verbal e doloroso, localizando os estímulos, movimentando o lado $\mathbf{E}$ espontaneamente e sem esboço de movimentos à D. Permaneceu ainda entre sonolência e torpor durante os dias subsequentes. Foi necessária traqueostomia e recebeu alta do CTI no dia 20-11 com o seguinte quadro clínico: consciente, afásica, comunicando-se por gestos, parecendo reconhecer as pessoas, hemiplegia D maciça proporcionada e completa, isocórica, fotorreagente, oculomotores normais, nuca livre. Manteve-se consciente até o dia da alta, em 02-01-91. Paciente com marca-passo por cardiopatia chagásica crnôica.

Caso 4 - AHT, 74 anos, feminina, parda. Sofreu icto cerca de 24 horas antes da internação, em 22-04-91. A TCC, realizada no dia do icto, mostrava infarto no território da artéria carótida interna $E$, com pequeno efeito de massa. Exame: PA: 13x8, consciente, um pouco sonolenta, isocórica, déficit motor maciço a D, afásica, respiração normal. Exame cardiológico normal. Manteve-se estável até o dia seguinte. No dia 24 entrou em estado torporoso, respondendo mal a estimulação verbal, respiração tipo Cheyne-Stokes, pupila $\mathbf{E}$ discretamente maior do que a $\mathrm{D}$, sendo administrado manitol. Não repetiu TCC por problemas técnicos. As 19:00 horas' mostrava-se com hipertonia em extensão bilateral, pupila $E$ maior que a $D$, sem resposta a estímulos verbais. Realizada lobectomia temporal E. Imediatamente antes da cirurgia a paciente estava em coma, com hipertonia em extensão bilateral, pupilas dilatadas (a $\mathbf{E}$ pouco maior que a $\mathrm{D}$ ), sem fotorreação. No dia seguinte permanecia com pupilas midriáticas (a E pouco maior que a D), sem fotorreação, com hipertonia em extensão bilateral, bradipnéia, sem o respirador. As 23:00 horas entrou em midríase fixa bilateral, sem qualquer reação motora. óbito no dia 28-04-91.

Caso 5 - JPN, 60 anos, branco, masculino. Paciente com hipertensão arterial, admitido em 09-05-91 com quadro de acidente vascular cerebral instalado há 60 horas. Consciente, relativamente orientado, com hemiplegia $\mathrm{E}$ maciça e completa. TCC: extenso infarto fronto-tèmporo-parietal D, com moderado efeito de massa. Medicado com manitol e dexametasona. Na noite da internacão foi transferido para o CTI em estado torporoso, necessitando de monitorização. No dia seguinte permanecia torporoso, com o mesmo déficit motor a E. No dia 11 entrou em coma, reagindo em hipertonia nos membros inferiores, discreta anisocoria (pupila $D$ maior que a E), sem responder aos comandos verbais. TCC: grande efeito de massa do infarto cerebral D. As 15:00 horas foi submetido a lobectomia temporal D. No dia seguinte permanecia inconsciente, não abrindo os olhos aos estimulos, mas isocórico e com fotomotores presentes. No dia 13 apresentava movimentos espontâneos à $D$, isocórico, fotorreagentes. Melhora gradual e mais ou menos rápida recebendo alta, do CTI no dia 18 e do hospital no dia 28-05-91, consciente, comunicando-se pela fala, isocórico, com hemiplegia $\mathbf{E}$ maciça.

Caso 6 -- LCP, 48 anos, branco, masculino. Admitido em 27-08-91 com quadro de hemiplegia D completa e afasia, instalado há 60 horas. Exame: PA: 13x8, consciente, isocórico, fotomotores presentes, nuca livre, carótidas normais e sem sopros, hemiplegia $D$ e afasia. Cardiologicamente normal. TCC: infarto têmporo-parietal E, com pequeno efeito de massa o paciente permaneceu consciente mas com o mesmo deficit até o dia 30 quando, às 16:30 horas, desenvolveu quadro abrupto de perda de consciência e hipertonia em extensão bila-

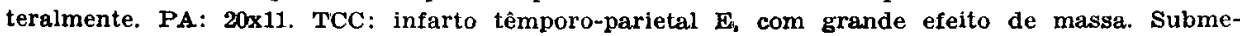
tido imediatamente a lobectomia temporal $E$. No dia seguinte permanecia em coma, com midríase bilateral, sem reação fotomotora, em apnéia e com respiração controlada. óbito no dia 31 às 23:00 horas. Este paciente foi medicado nos três primeiros dias da internação com Rheomacrodex e nimodipina. A angiografia mostrou obstrução parcial da artéria cerebral média $\mathbf{E}$ e dos ramos rolândico e parietal.

Caso 7 - AM, 66 anos, branco, masculino. Há 2 horas desenvolveu quadro de dor de cabeça, vômitos e instalou-se hemiplegia E. Exame: PA: 13x7, consciente, mas não obedece a comandos, isocórico, fotomotores presentes, desvio dos olhos para a D, nuca livre, hemiplegia E maciça. Exame cardiológico normal. Medicado com Rheomacrodex e ticlopidina. TCC: pequenas áreas hipodensas e apagamento de sulcos corticais na regiâo fronto-parietal $D$. No dia seguinte permanecia com quadro clínico estável mas, à tarde, apresentou piora do nivel de consciência tornando-se torporoso, com respiração tipo Cheyne-Stokes mas lainda isocórico e fotorreagente. TCC: extenso infarto fronto-têmporo-parietal, comprometendo principalmente o lobo temporal, com grande efeito de massa. Submetido a lobectomia temporal $D$, à noite deste día. No dia seguinte mostrava-se com reação de despertar, atendendo a comandos verbais, isocórico e fotorreagente, com a mesma hemiplégia E. Recebeu alta do CTI no 4\% P.O. e alta hospitalar 10 dias após, consciente, lúcido e com hemiplegia macica E. 
Caso 8 - MBS, 57 anos, branca, feminina. Há dois dias (13-11-91) sofreu paralisła súbita do lado $\mathbf{E}$ do corpo, com vômitos e cefaleia. Piorou hoje, estando torporosa, isocórica, fotorreagente, com hemiplegia maciça $\mathrm{E}$ e hipertonia. PA: 13x8. TCC: extenso infarto hemorrágico fronto-têmporo-parietal $D$ e moderado efeito de massa. Portadora de doença de Chagas. Medicada com manitol e dexametasona. No dia seguinte a internação entrou em coma, com abundante secreção brônquica, respirạăo tipo Cheyne-Stokes, hipertonia em extensåo $\mathbf{E} \theta$ discreta anisocoria. Foi operada (lobectomia temporal D). Melhorou do nível de consciência, mas manteve a atitude de hipertonia em extensão no lado hemíplégico por vários dias. Apresentou compliaaçôs respiratórias e só recebeu alta hospitalar 1 mês após, consciente, lúcida e com hemiplegia $\mathbf{E}$ maciça.
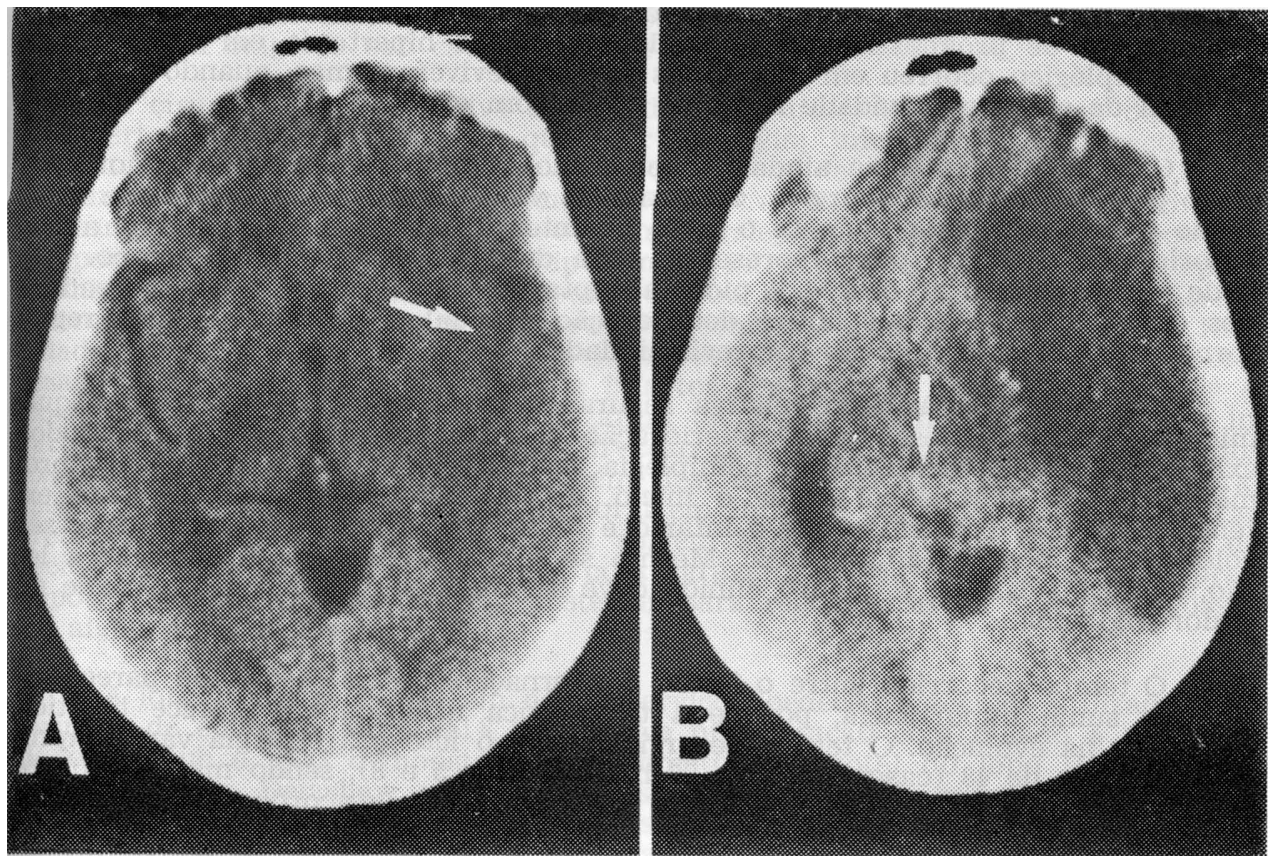

Fig. 1. Caso 2. Em A, TCC inicial, 4 horas apbs o icto, mastrando pequeno efeito de massa com assimetria da cisterna silviana, menor a esquerda (seta). Em B, TCC após 48 horas, pré-operatória, com área de infarto bem caracterizada e grande efeito de massa, com pinosl desviada em $11 \mathrm{~mm}$ (seta) e apagamento das cisternas.

\section{RESULTADOS}

Dos 8 casos submetidos a lobectomia temporal descompressiva, 6 tiveram evolução favorável (75\%). Um paciente (caso 4) que faleceu estava em estiado mais grave no momento da cirurgia, já com midriase bilateral e reação de hipertonia em extensão bilateral. A cirurgia não modificou a evolução, permanecendo no mesmo estado prévio e, a seguir, entrou em morte cerebral. O outro caso fatal (caso 6), o mais jovem do grupo, estava estável, consciente

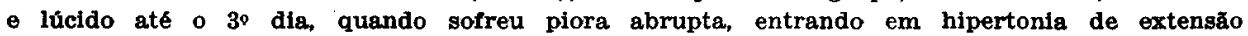
bilateral e coma, năo havendo melhora com a intervenção cirúrgica.

Os 6 sobreviventes permaneceram com déficit motor severo até o momento da alta. Com exceção dos casos 3 e 7 , os outros tiveram pós-operatório complicado, várias intercorrências e internação acima de 20 dias. Os 4 pacientes com lesão do hemisfério náo dominante (casos 1, 5, 7 e 8) estão lúcidos e até organizando seus negócios. Os 2 com hemiplegia direita (casos 2 e 3) mantêm severo deficit de linguagem, com afasia tipo misto, predominantemente motora. O resultado das avaliaçőes neuropsicológica e fonoaudiológica serão apresentados posteriormente. 
A indicação da cirurgia descompressiva é resultante da observação do quadro clínico e do aspecto tomográfico 10,12 .

A indicação clínica se baseia principalmente na piora do estado neurológico, peia apresentação de sinais de deterioração de funções do tronco cerebral, na sua clássica evolução crânio-caudal ${ }^{9}$. Todos os nossos pacientes estavam inicialmente conscientes ou com leve comprometimento da consciência. Sua piora foi elemento clínico fundamental que, por si só, chamou a atenção para a gravidade do quadro. A alteração do padrão respiratório, com apresentação do ritmo de Cheyne-Stokes, foi outro sinal significativo. Anisocoria ocorreu frequentemente, mas em um dos casos que perdemos, só apareceu tardiamente. Miđríase bilateral mostrou ser de prognóstico fatal em dois casos. A hipertonia em extensão do lado hemiplégico ocorreu em dois casos que sobreviveram mas, quando bilateral, foi sinal de enorme gravidade nos dois casos com evolução fatal.

Com esta experiência ainda pequena, não se pode definir qual o momento exato para intervenção cirúrgica, do ponto de vista clínico. Há receio de se estar sendo precipitado, mas corre-se o risco de intervir tardiamente. Aguardar a anisocoria, por exemplo, pode representar uma desnecessária perda de tempo. Isto pode ter acontecido no caso 4. A piora abrupta do caso 6 não obedeceu ao padrão dos outros casos em que houve deterioração gradual e, portanto, foge à regra, tornando-se um exemplo de imprevisibilidade.

Pode-se antecipar com relativa segurança quais os casos de infarto isquêmico que poderão desenvolver deterioração clínica, como nestes 8 casos. Todos exibiam padrão semelhante de hemiplegia maciça e, a maioria, algum comprometimento de consciência desde o início, denunciando lesão provavelmente extensa. Se a TCC confirmar que o infarto é realmente grande, o paciente deve ser colocado em observação rigorosa. Mesmo se a TCC falhar em demonstrar a lesão, como no caso 1 , o quadro clínico deve ser suficiente para tomar atitude de maior vigilância.

O tempo decorrido entre o icto e a internação, nestes casos, não parece ter sido de suma importância, porque a terapêutica utilizada não parece ter tido influência na evolução. $O$ tempo decorrido entre o icto e a cirurgia variou aproximadamente de 48 (casos 2 e 7 ) a 96 horas (casos 5 e 8), sendo mais frequente em média de 72 horas (casos $1,3,4$ e 6), o que está de acordo com outras referências 2,10 .

A idade dos pacientes não se mostrou fator apreciável, já que os óbitos ocorreram nos pacientes de idades extremas neste grupo, o mais jovem (48 anos) e o mais idoso ( 74 anos). Entretanto, para ambos, pode haver explicações que não seja o fator idade: o mais jovem teve piora abrupta inexplicável e o mais idoso estava muito mais grave que os outros quando foi operado. A hipertensão arterial ocorreu em apenas 2 casos e o diabete melito não estava presente neste grupo. Doenças e complicações sistêmicas apenas dilataram o tempo de internação e não parecem ter tido influência na evolução do quadro neurológico dos pacientes.

A TCC é método diagnóstico imprescindivel para exclusăo de hemorragia intraparenquimatosa. Em infartos extensos costuma ser demonstrativa desde as primeiras horas, mas há exceçōes como no caso 1. Sua repetição deve ser compulsória, não estando ainda estabelecida com que frequência. Aguardar a piora clínica, como fizemos, pode incorrer em atraso na intervenção cirúrgica. Outro problema reside na definição dos parâmetros tomográficos que possam servir como indicadores da cirurgia. Em nossos casos, talvez pela pequena mostra, não chegamos a definir medidas e limites 11,12 , apenas valorizamos desvios maiores da linha média, apagamento de sulcos e cisternas e, principalmente, a visualização da cisterna peripeduncular (Fig. 1B). Por motivos econômicos, não repetimos tantas TCC como seria desejado, principalmente nos pós-operatórios e na evolução posterior. Por isso, reconhecemos que há bastante limitação em nossa experiência com a TCC e aguardamos estudos posteriores que complementem este assunto. 
Nos casos apresentados não parece ter havido influência da medicação prévia à cirurgia. Em 5 casos usou-se o dextran, que era rotina em nosso Serviço, e em 2 dexametasona, com ou sem nimodipina associada (apenas naqueles casos mais precoces). Em 4 casos, quando se verificou a piora clínica e tomográfica, introduziu-se manitol, que não parece ter modificado a evolução natural. Nenhum paciente recebeu heparina, devido à extensão da lesão, nem agentes trombolíticos, que não eram disponíveis em nosso Serviço.

Em nenhum caso foi instalada monitorização da pressão intracraniana, que taivez fosse elemento importante a ser levado em conta na conduta, como acontece em traumatismos cranioenfecálicos 7. Em apenas um paciente houve vigorosa tentativa de prevenir a hipertensão intracraniana com entubação, respiração controlada e hiperventilação. Os outros casos estavam sendo cuidados nas enfermarias, com oxigênio sob catéter nasal e respiração espontânea.

A cirurgia descompressiva é procedimento utilizado na tentativa de aliviar a hipertensão intracraniana de qualquer etiologia; inicialmente foi preconizada para tumores cerebrais inacessiveis, depois estendida a outras patologias, principalmente nos traumatismos cranioencefálicos. Kjellberg e Prieto 5 citam que a cirurgia descompressiva começou com Horsley, que realizou a craniectomia temporal direita, para alívio de hipertensão intracraniana. Alguns anos depois, Cushing sugeriu a variante subtemporal uni ou bilateral, que se tornou clássica. Outros autores se sucederam, descrevendo técnicas variadas com a mesma finalidade. Botterell foi o primeiro a realizar ressecção de parte do tecido cerebral lesado. Duncan et al. $3^{3}$ aplicaram-na a infartos cerebelares. Rengachary et al.10 e, posteriormente, Delashaw et al. ${ }^{2}$ utilizaram técnica de hemicraniectomia para infarto massivo hemisférico, com preservação do tecido cerebral, com resultados Excelentes. Kondziolka e Fazl ${ }^{6}$ também descreveram bons resultados com craniectomia descompressiva fronto-temporal em infartos com efeito de massa. Entretanto, as técnicas descompressivas sofrem sempre a crítica da extrusão do tecido cerebral ao nível da cirurgia, com agravamento da lesão 1. Também pressupõem a realização de cirurgia posterior para recolocação do fragmento ósseo ou algum tipo de plástica.

A lobectomia temporal em nosso Serviço obedeceu à técnica utilizada para cirurgia de epilepsia temporal 8 Não houve preocupação com o lado a ser abordado, porque a TCC demonstrou comprometimento total do lobo temporal em todos os casos. Delashaw e seu grupo 'a só realizaram hemicraniectomia descompressiva sobre o lado não dominante, justificando-se pela qualidade de vida dos sobreviventes. A experiência com epilepsia demonstra que a lobectomia temporal só interfere com a memória (e isto apenas em alguns casos) e não com outras funções corticais. Assim, não acreditamos que a lobectomia tenha acrescentado déficits aos nossos pacientes. Todos que receberam alta hospitalar estavam com sequelas importantes, mas o fato de sobreviverem nos satisfez, independentemente da qualidade de vida. Acompanhamento posterior, para verificar o grau de recuperação, terá peso importante nesta questão. Comparar a casos não operados talvez seja difícil já que, com o efeito benéfico das cirurgias descompressivas, a não indicação cirúrgica pode se tornar problema ético.

A lobectomia temporal apresenta dois pontos vantajosos em relação às descompressivas sem ressecção de tecido. Em primeiro lugar está o fechamento dos planos com facilidades e a reposição do osso, evitando intervenções reparadoras posteriores. $\mathbf{E}$, ainda, a eliminação pela abordagem direta do elemento compressivo, o uncus e outras estruturas mesias do lobo temporal.

O resultado obtido com a lobectomia temporal descompressiva, com sobrevivência de $75 \%$, é estimulante. Dos dois insucessos, pelo menos um pode ter sido devido a fator provavelmente contornável com indicação cirúrgica mais precoce. Experiências em outras patologias parecem corroborar o efeito salva-vidas deste procedimento cirúrgico 9 . Entretanto, como a amostra é reduzida não queremos tirar conclusões definitivas e precipitadas. Mas, os resultados iniciais da lobectomia temporal descompressiva justificam uma cuidadosa atenção da comunidade neurológica e neurocirúrgica, estimulando a produção de estudos melhor controlados. 


\section{REFERENCIAS}

1. Cooper PR, Hagler H, Clark WK, Barnet P. Enhancement of experimental cerebral edema after decompressive craniectomy: implication for the management of severe head injuries. Neurosurgery 1979, 4:296-300.

2. Delashaw JB, Broaddus WC, Kassell NF, Haley EC, Pendleton GA, Vollmer DG, Maggio WW, Grady MS. Treatment of right hemispheric cerebral infarction by hemicraniectomy. Stroke 1990, 21:874-881.

3. Duncan GW, Parker SW, Fisher CM. Acute cerebelar infarction in the PICA territory. Arch Neurol 1975, 32:364-368.

4. Fisher M. Clinical pharmacology of cerebral ischemia: old controversies and new approaches. Cerebrovasc Dis 1991, 1 (Suppl 1):112-119.

5. Kjellberg RN, Prieto A Jr. Bifrontal decompressive craniotomy for massive cerebral edema. $\mathrm{J}$ Neurosurg 1971, 34:448-493.

6. Kondziolka D, Falz M. Functional recovery after decompressive craniectomy for cerebral infarction. Neurosurgery 1988, 23:143-147.

7. Nussbaum ES, Wolf AL, Sebring L, Mirvis S. Complete temporal lobectomy for surgical resuscitation of patients with transtentorial herniation secondary ot unilateral hemispheric swelling. Neurosurgery 1991, 29:62-66.

8. Olivier A. Commentary: cortical resections. In Engel $J$ (ed): Surgical treatment of the epilepsies. New York: Raven Press, 1987, p 405-416.

9. Plum F, Posner JB. The diagnosis of stupor and coma. Ed 3. Philadelphia: FA Davis, 1982.

10. Rengachary SS, Batnitsky S, Morantz RA, Arjunan $K$, Jeffries B. Hemicraniectomy for acute massive cerebral infarction. Neurosurgery 1981, 8:321-328.

11. Ropper AH. Lateral displacement of the brain and level of consciousness in patients in acute hemispheral mass. N Engl J Med 1986, 314:953-958.

12. Ropper AH, Gress DR. Computerized tomography and clinical features of large cerebral hemorrhage. Cerebrovasc Dis 1991, 1:38-42.

13. Ropper AH, Shafran B. Brain edema after stroke: clinical syndrome and intracranial pressure Arch Neurol 1984, 41:26-29. 